\title{
Design and Development of Hexapod using Nitinol Actuator Wire
}

\author{
Asadullah Rahoo, Abdullah Qazi, Irfan Ahmed Halepoto*, Pirya lohana, Ameet Kumar and Bushra \\ Shaikh \\ Department of Electronic Engineering, Mehran UET, Jamshoro, Pakistan; irfan.halepoto@gmail.com
}

\begin{abstract}
Objectives: This research work involves the design and implementation of Hexapod robot which is a small, inexpensive, six-legged robot. Methods/Statistical Analysis: Hexapod robots from decennium have gained considerable attention in various research and development sectors. This research work involves the design and implementation of Hexapod robot which is a small, inexpensive, six-legged robot intended to replace huge and heavy robotic that are used in space applications, industries for lifting purpose containing solenoids and servo motors. Findings: Initial stage of research work involves design and construction of the structure of Hexapod. Plastic material is used for the construction of body and a Nitinol actuator wire is used to drive the Hexapod's legs. The final stage involves Hexapod interfacing with Arduino and HBridge module for supply to achieve proper locomotion of the Hexapod. Specifically, real element like mechanical structure, legs arrangement, impelling, payload, movement condition and waking walk are considered in proposed system design. In this work, a novel robot is constructed and found that conservative, and lightweight Hexapod robot demonstrates guarantee for use in space, therapeutic, and other large scale robotic applications. Application/Improvements: Due to the unique actuating mechanism of nitinol wire the proposed and developed model has the impact significance in many application fields.
\end{abstract}

Keywords: Arduino, Flexinol Wire, Hexapod, Stiquito

\section{Introduction}

Concept of small inexpensive and less complex hexapod robots is getting ore attraction in research and development as bulky and complex hexapod robots have limited applications in robotic design. The hexapod robots concept was introduced by Johnathan $\mathrm{W}$ Mills and was named as Hexapod Stiquito using nitinol wire. In the initial hexapod design, the control circuitry for hexapod legs movement was inadequate as Stiquito was powered by 9 volt batteries continuously which could burn Flexinol wire. Considering this, James M Conrad made the advancement in Stiquito by designing a PCB board to control legs of Stiquito using MSP430 microcontroller. The developed Stiquito has still some issues in PCB design but there was no any Joint Test Action Group (JTAG) interface. The JTAG power option was selected through the jumpers but no speed controls were there to include the new features of the microcontrollers as input/output parameters to be reordered. It also has code consist of many lines. The aim of this work is to analyze how nitinol wire actuator can be controlled by different way ${ }^{1}$ by using Arduino to control legs of Stiquito. The proposed design model is shown in Figure 1. The power supply of $12 \mathrm{~V}$ is given to the legs of hexapod according to their power handling capability and is regulated through Arduino. The locomotion of hexapod is controlled by Arduino program using different Gait methods. As a result, the locomotion of robot can be seen easily. Different sensors can be used according to different applications which make enhancement in the field of miniature robots. The speed of robot can also be changed by increasing or decreasing delay in program by using different algorithms. With the advancement of material technology, it is possible to design more compact and light weight robotic system. However, the presence of standard actuators, such as servo motors and

*Author for correspondence 
hydraulic cylinders limits this reduction of size and complexity of robots 2 .

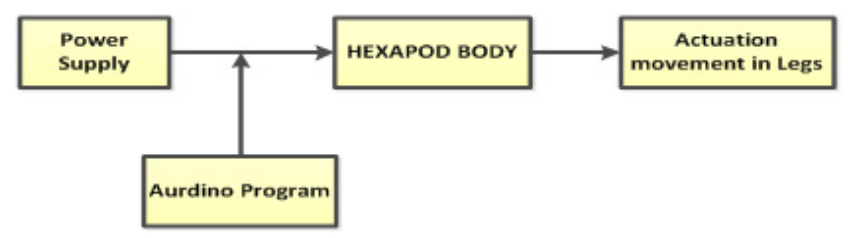

Figure 1. Block diagram of Hexapod Robot.

To replace these standard actuators and motors we have developed a structure and have used nitinol wire as an actuator to loco mote that structure. The nitinol wire have been used for the movement of robot to observe and analyze how the nitinol wires works, what are its different applications in the field of robotics and its properties. One of the major applications of six legged robots is its utilization as search and rescue robot. The rest of paper proceeds as follows: In Section 2 materials and methods are discussed to characterize the nitinol wire functionality. The proposed model design structure of Hexapod Robot is developed in Section 3. The physical implementation of Hexapod Robot is presented in Section 4. The experimental analysis is carried out in Section 5. In section 6 , the research work is concluded.

\section{Material and Methods}

Actuator wire is made from an alloy of nickel-titanium that why so called as Nitinol, and marketed under a number of brand names including Flexinol, Bio Metal and Muscle. It is thin, highly processed strand of a nickeltitanium alloy that is why it is also called as Nitinol wire. This small diameter wire contracts like muscle when electrically driven ${ }^{3}$. It comes in eleven sizes and two transition temperatures as mention. Both the withdrawal and unwinding are for all intents and purposes quick with the unwinding of wire ${ }^{4}$. Flexinol is easily stretched by a small force at room temperature. When it conducts an electric current, the wire warms and changes its shape by stretch and changes to a much harder shape that proceeds to the "unscratched shape at low temperature by using some stressing force. Cooling time at High Temperature (HT) and Low Temperature (LT) is illustrates in Table 1.

Wires abbreviate long with a usable measure of force. It can be shortening by up to 3 to $5 \%$ range to keep running many cycles with exceptionally solid execution ${ }^{5}$. Jonathan W Mills first gave the idea and built structure of hexapod named Stiquito using nitinol wire. Different materials were used for the structure to make it cost efficient. Main body was made up of plastic material with that plastic body legs, nitinol wire and control circuitry was attached. A tube of copper was used as a conducting bus through that supply can be given to all legs. Legs were made of musical wire of smaller diameter $(0.020$ inch) as it is more flexible due to more flexibility it cannot carry more load. Nitinol wire of only one diameter was used. To make locomotion of robot more efficient smaller diameter can be used as wire of different diameters has different cooling time at different temperature. Stiquito is hard-wired to stroll in a tripod step. Different steps can be hard-wired by evacuating the body creases and re-wiring the control wires. Every leg might be wired independently for more complex walks. Hexapod Stiquito has four different walking methods also known as gait methods: (a) Tripod Gait, (b) Pace Gait, (c) Caterpillar Gait, (d) Independent Gait.

(a) Tripod Gait: In tripod Gait method, three legs of hexapod's are in contact with ground all the times: the back and front legs on one side and the middle leg on the opposite side ${ }^{6}$. The point of the center leg indicates which side is up and which side is down. It accomplishes the forward movement by pushing those feet in reverse against the ground while the other feet advance through the air. At that point the hexapod moves its weight to the next three feet and advances similarly. By incessantly moving its weight by utilizing the center legs, it propels towards raising feet it strolls forward.

(b) Pace Gait: For the motion in straight direction three legs of same side are powered at time and three legs

Table 1. Characteristic and properties of nitinol wire

\begin{tabular}{|l|l|l|l|l|l|}
\hline $\begin{array}{l}\text { Wire diameter } \\
\text { inches }(\mathbf{m m})\end{array}$ & $\begin{array}{l}\text { Resistance pull } \\
\text { force (ohms/ } \\
\text { meter) }\end{array}$ & $\begin{array}{l}\text { Pull force } \\
\text { pounds } \\
\text { (grams) }\end{array}$ & $\begin{array}{l}\text { Current contact } \\
\text { in 1 second ( } \\
m A)\end{array}$ & $\begin{array}{l}\text { Cooling time } \\
\text { “LT” wire } \\
\text { (seconds) }\end{array}$ & $\begin{array}{l}\text { Cooling time } \\
\text { “HT” wire } \\
\text { (seconds) }\end{array}$ \\
\hline $0.004(0.10)$ & $3.2(126)$ & $0.31(143)$ & 200 & 1.1 & 0.9 \\
\hline $0.006(0.15)$ & $1.4(55)$ & $0.71(321)$ & 410 & 2 & 1.7 \\
\hline
\end{tabular}


2, 4, 6 on the other side helps the robot pushing in forward direction. After some delay when wire legs of other side are powered and legs 1, 3, 5 helps the robot pushing in forward direction.

(c) Caterpillar Gait: Envision legs of the robot are marked in such a way that legs along the left side are named 1, 3 and 5, and the legs on the right hand side are named 2, 4 and 6. Front two Legs on either side name 1 and 2 are raised and swung forward, while legs on 3, 4, 5 and 6 backing and push the body advances. Legs 5 and 6 at that point swing forward, while the rest sponsorship and push the body forward. Legs 3 and 4 then swing forward, while the rest sponsorship and push progresses.

(d) Independent Gait: In independent Gait step has no altered or intermittent stride; even its strolling instrument is dictated by the territory and state of a machine. This system is exceptionally entangled however gives the robot a more adaptable stride to better cross confused territory.

\section{System Design}

Early Hexapod with actuator Nitinol wire was named as Stiquito; it was a small inexpensive robot with simple operating principles and control gait. The Stiquito was designed according to actuator characteristics i.e., main structural part as the body, legs, controller, power bus and the actuator. Our focus was to design a hexapod with characteristic different from the Stiquito as it is trade name of Hexapod using Nitinol. We have labeled our robot as Spiderbot as shown in Figure 2, which is designed on the principle of Stiquito but it differs significantly as main part of Spiderbot are body, legs, power bus, Arduino and the actuator. The whole structure depends how it gets the strength and locates the attachment point for actuating wire and legs. The material used for body is of plastic which is of rectangular shape. Usually the body is made of metallic or steel material but to prevent from electric shock and high complexities engineers and designer preferred plastic and even wood as it requires using the light body material so that actuator can work properly. Secondly the legs are made up of stainless steel material and it performs the following functions:

1. Legs support the total weight of hexapod.

2. The stainless steel provides the precise amount of recovery force to nitinol wire.

3. All of the legs share the normal electrical power connected with the power bus.

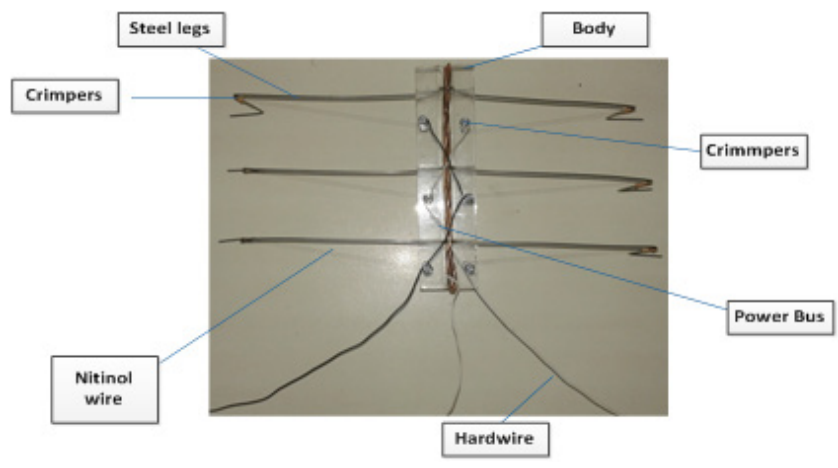

Figure 2. Structure of Hexapod.

In electrical power distribution; a bus bar (power bus) is combination of two copper wire twisted together to conduct electricity ${ }^{7}$. Its principle object is to direct a considerable current of power, and not to work as an auxiliary part. Bus bars can decrease the power losses by means of diminishing the corona Effect. This is on the grounds that bus bars have greater surface regions contrasted with wires. The material piece and cross-sectional size of the bus bar decide the greatest measure of current that can be securely carried. The Controller used for nonstop movement of spiderbot is Arduino which is an open-source prototype. The actuator wire used is Nitinol wire (trade name flexinol wire). Flexinol actuating wires are of little diameter wires which contract like muscles when are derived electrically. To regain the original size of the wire bias force or recovery force is needed. Flexinol was attached with the legs and power bus with the help of copper tape.

Arduino boards can read inputs like light sensing, a finger on a catch, or a twitter message and transform it into an output, actuating an engine, turning on LED etc. Arduino comes in various microcontroller board but here ATmega 128 is being utilized which has 54 advanced information/yield pins (of which 14 can be utilized as PWM yields), 16 simple sources of info, 4 UARTs (equipment serial ports), a $16 \mathrm{MHz}$ gem oscillator, a USB association, a power jack, an ICSP header, and a reset button. It has been powered by use of $\mathrm{H}$-bridge which is use for providing exact amount of voltage to the actuating wire. Arduino is being used for providing continuous voltage and controlling the gait movement of spiderbot.

\section{Design Implementation}

The all parts of spiderbot are integrated together as a one system including the construction of body with the plas- 
tic thickness of $1 \mathrm{~cm}$ to the length of 6 inch and width of 2 inch. The interfacing focuses for the legs and actuators are a progression of gaps and furrows that are bored, sawed, and cut into the body. The legs are amassed in sets from three $180 \mathrm{~mm}$ lengths of stainless steel. The stainless steel legs perform three tasks. Begin the process by cutting three $180 \mathrm{~mm}$ lengths of stainless steel then bend it, try not to twist the wires too far or they may split or break. The apex which forms the V-clamp ought to be adjusted, not sharp. Cut a two or three copper wire and fixed them all and heat the copper wire so to make a straight gape of $4 \mathrm{~mm}$ in between the plastic body and adjust the legs in way to form the V shape. Turn the body over and permanently clamp the power bus, simultaneously attaching the legs, by spreading each leg in the pair outward by hand, at the same time pulling upward on the legs. When the legs are almost horizontal, grasp each leg in turn with the needle nose pliers and firmly bend it downward, while continuing to pull outward, until the leg snaps into the clip groove. At this point the power bus should be securely clamped into place. Check to ensure that it will not touch any of the body crimps. The electrical connection between the power bus and the legs should be less than $2 \mathrm{ohms}$. Adjust the legs so that they are in a plane horizontal with the bottom of the body, and parallel to each leg. It is important to bend the stainless steel at $90^{\circ}$ up about $80 \mathrm{~mm}$ from the edge of the body ${ }^{8}$. The Flexinol wire changes over the heat induced by an electric current into mechanical movement supplanting stepper motors, screws, and different parts generally expected to make a leg move. The mechanical movement is due to the changes in the crystalline structure of nitinol. The nitinol diameter defines the electric current and resistance values.

\section{If we consider nitinol wire of $0.006^{\prime \prime}$ diameter}

$$
\text { then it reference value is } 1.4 \text { per inch and }
$$
current need to drive the wire is $410 \mathrm{~mA}$ and if we take 0.004 "diameter of the wire then the resistance value is 3.2 per inch and current need to drive the wire is $200 \mathrm{~mA}$. Here we have used $160 \mathrm{~mm}$ for three stainless steel wire with diameter of $0.006^{\prime \prime}$ and $0.004^{\prime \prime}$ so each has $80 \mathrm{~mm}$ of the nitinol wire. The crimps are made up of aluminum tube to make something rigid or stiff. The Nitinol wire is attached to the legs in such a way that the legs move properly and fast, so the nitinol wire is knotted on the leg properly. The nitinol wire should be knotted at the angle of $90^{\circ}$ with the legs, if knotted angel is less in $90^{\circ}$ at bottom of the legs, the legs will move in and out. Once our body, legs, power bus and the nitinol wires are attached together with $0.006^{\prime \prime}$ diameter nitinol wire, the voltage and resistance value of wire can be calculated as: Voltage $=12 \mathrm{~V}$ the current required is $=410 \mathrm{~mA}$ The resistance value of the wire $=\frac{1.4 \Omega}{\text { inch }}$ so we are using $\mathbf{8}$ inch then it is $1.48=11.2$

But we have used $22 \Omega$ or we can used two $10 \Omega$ resistor in series, as to get the exact value of current so we drive our legs.

First it is necessary to whether check single leg is working properly or the nitinol is not burn out so we can use one $22 \Omega$, 5 watt power as there heat sink capability is good and do not burn early. Now apply the ground to the power bus and the positive supply of $12 \mathrm{~V}$ to the one terminal of resistor and other terminal output to the screw on which the nitinol wire is connected. This will provide the supply about half second to check wire and leg movement. If it leg is not moving the time can be increased more than half second, this will burn the nitinol wire ${ }^{9}$. To move spiderbot continuously the Arduino is used due to simplicity and easy to program, but continuously supplying power to spiderbot for its movement it can burn out the flexinol wire. For this exact voltage and current values are defined for every wire diameter as illustrated in Table 1. There are total 54 digital pins of Arduino platform, out of which 14 pins are PWM, but we have only used six pins from 22 to 27 just to provide inputs to six legs to power them turn on and turn off with defined time (i.e., on time $=500 \mathrm{~ms}$ and off time $=1000 \mathrm{~ms}$ ) as shown in Figure 3. The off time is larger because the nitinol need to deform to its original shape. The dual H-bridge motor driver module known as Power Bridge is used to drive the suitable power using $V_{\text {in }}$ pin to supply $12 \mathrm{~V}, 5 \mathrm{~V}$ pin will drive the Arduino and $12 \mathrm{~V}$ will drive the jumper. The legs are programmed to move in Tripod fashion into as 3 pair of legs i.e. $(1,4,5$ and 2, 6, 3) as one pair will be ON and OFF with the defined time delay. Corresponding to this when first pair will move at defined time the second pair will be in stop mode as shown in flow chart in Figure 3. The graph of distance covered by Stiquito robot is shown in Figure 4. The testing circuit of nitinol wire (Flexinol) and graph of distance cover by Spiderbot are shown in Figures 5 and 6 respectively. 


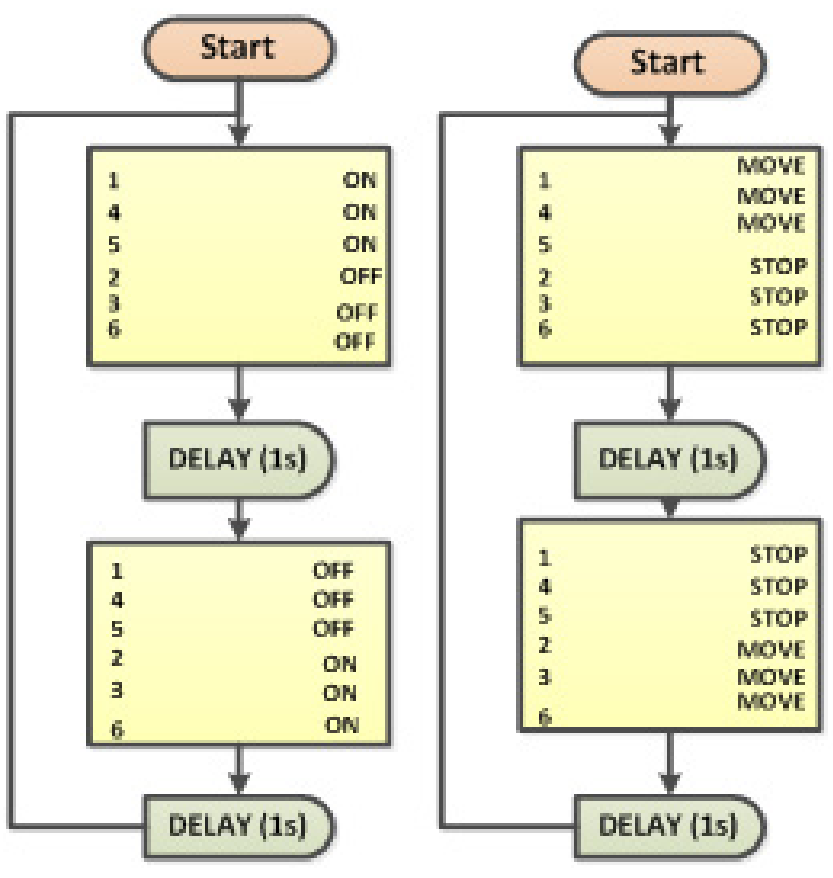

Figure 3. Digital pins pairing flow chart.

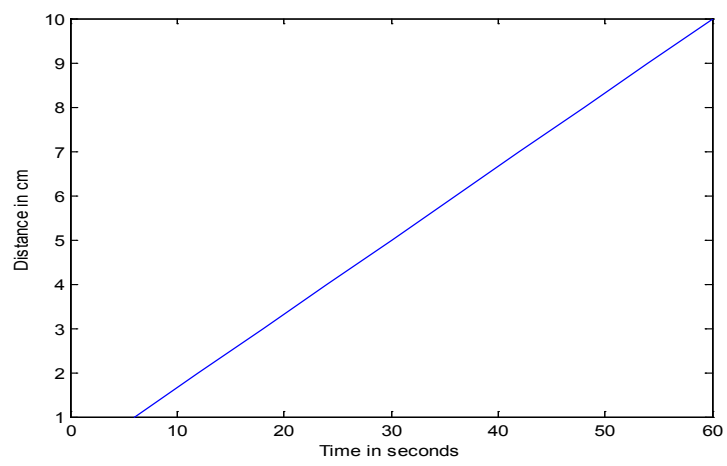

Figure 4. Graph of distance covered by Stiquito Robot.

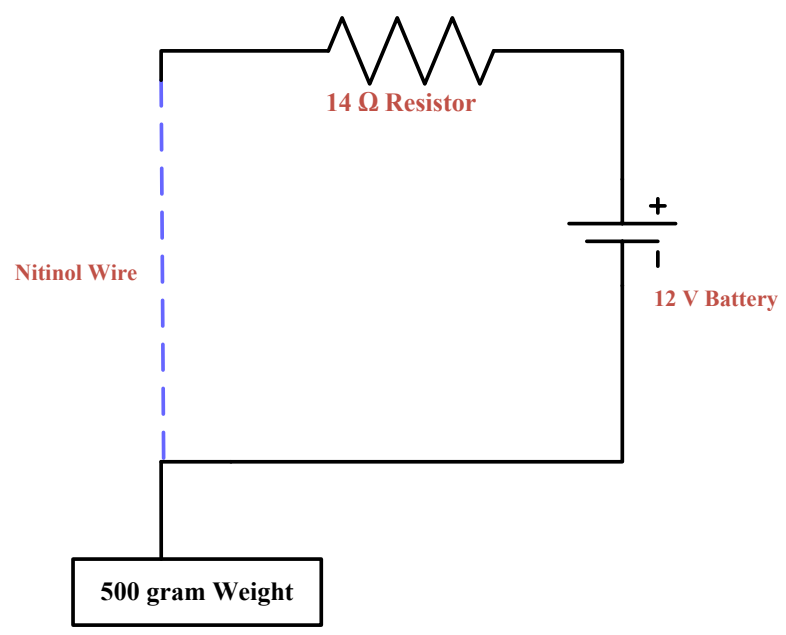

Figure 5. Testing circuit of nitinol wire (Flexinol).

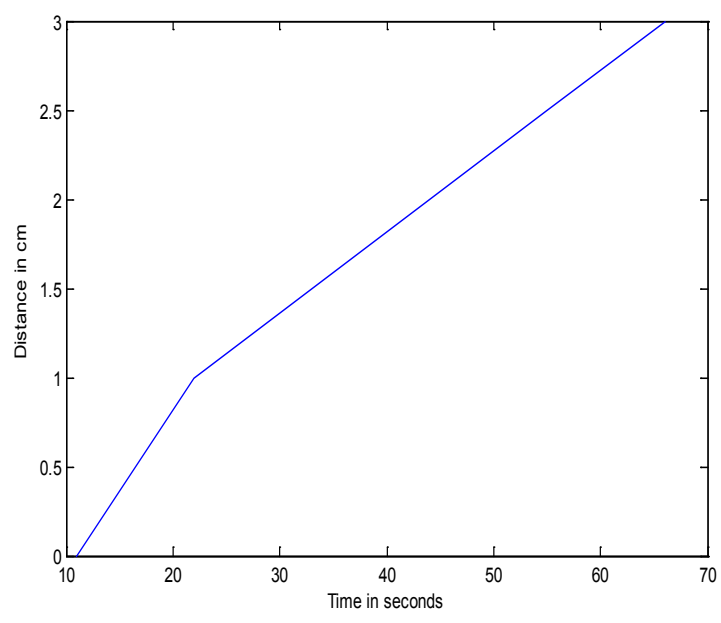

Figure 6. Graph of distance cover by Spiderbot.

\section{Experimental Analysis}

Once the hexapod is developed and designed it has been analyzed with 0.004 " moving diameter with the recorded speed of $10 \mathrm{~cm}$ per minute. On other hand when larger diameter of 0.006 "is moved then the speed of hexapod is reduced to $3 \mathrm{~cm}$ per minute. So it can analyze that when diameter of the Flexinol is increased the weight caring capability also increases but the speed of robot decreases. Also if wire of smaller diameter is used the weight caring capability decreases on other hand the speed of robot increases. This is due to the cooling time of large diameter is more as compare to cooling of small diameter as shown in Table. 1. So the speed of hexapod with larger diameter can be increased if we used spring in attached with actuating wire as the pulling force of the Flexinol wire. The pulling force of the spring and the actuator will easily deform and the speed of the robot will increase This will make able to pull large weight on other hand if want decrease the cooling time we have operate or run our robot in cool area or in room temperature atmosphere. Stainless is used so the robot should not have weight carrying problems

\section{Conclusion}

In this research work a smaller scale robotic system with different controlling method that are much lighter, and much more compact than existing systems is proposed which can retain the strength to perform the assigned task. In this work new methodology is used to reduce the 
size and weight of complex robotic system using of nitinol actuator wire by considering their capability to adopt a predetermined shape in a relatively simple manner, which results in a silent and smooth movement of hexapod in any direction.

\section{Acknowledgement}

Authors acknowledge the support of Mehran University of Engineering and Technology Jamshoro for providing necessary laboratory resources to conduct the research work.

\section{References}

1. Tucker SD, Conrad J, Rai Y. Design review for Stiquito PCB; 2010.

2. Halepoto IA, Shaikh MZ, Chowdhry BS, Uqaili MA. Design and implementation of intelligent energy efficient conveyor system model based on variable speed drive control and physical modeling. International Journal of Control and Automation. 2016; 9(6):379-88.

3. Blanco AG, Jiménez G, Moreno PO. Development of a functional neuromuscular stimulation system for inde- pendent ambulation of patients with a spinal cord injury. Engineering Letters. 2007; 15(1):73-81.

4. Available from: http://robotics.hobbizine.com/flexinol. html.

5. Technical characteristics of flexinol ${ }^{\circledR}$ actuator wires table of contents makers of dynamic alloys nickel - titanium alloy physical properties [Internet]. Available from: http://www. dynalloy.com/pdfs/TCF1140.pdf.

6. Stevanovi I. Development of a miniature robot based on experience that is inspired by nature. Proceeding of Applied Mathematics; 2013. p. 49-50.

7. Halepoto IA, Uqaili MA, Chowdhry BS. Least square regression based integrated multi-parametric demand modeling for short term load forecasting. Mehran University Research Journal of Engineering and Technology. 2014; 33(2):21526 .

8. Mills JW. Inexpensive technology lab exercises for grades 6-9. IEEE Twenty Fourth Annual Conference Proceedings in Frontiers in Education Conference; 1994. p. 218-22 .

9. Song H, Kubica E, Gorbet R. Resistance modelling of SMA wire actuator. International Workshop Smart Materials Structures \& NDT in Aerospace; 2011. p. 2-4. 